\title{
Increased Risk of Breast and Uterine Cancer Among Women With Ovarian Granulosa Cell Tumors
}

\author{
DIMITRIOS NASIOUDIS ${ }^{1}$, ELISE WILSON ${ }^{1}$, SPYRIDON A. MASTROYANNIS ${ }^{1}$, \\ GIOVANNI SISTI ${ }^{2}$, ASHLEY F. HAGGERTY ${ }^{1}$, EMILY M. KO ${ }^{1}$ and NAWAR A. LATIF ${ }^{1}$ \\ ${ }^{1}$ Division of Gynecologic Oncology, University of Pennsylvania Health System, Philadelphia, PA, U.S.A.; \\ ${ }^{2}$ Department of Obstetrics and Gynecology, University of Florence, Florence, Italy
}

\begin{abstract}
Background/Aim: We evaluated the incidence of uterine and breast cancer among women diagnosed with granulosa cell tumors (GCTs) of the ovary. Patients and Methods: The US Surveillance, Epidemiology, and End Results (SEER) database was accessed and patients diagnosed with a GCT and had a known follow-up between 1973-2014 were identified. Personal tumor history was extracted and patients with a previous or subsequent malignant breast or uterine tumor were identified. The expected incidence of breast and uterine cancer was calculated based on the U.S agespecific rate of breast and uterine cancer per 100,000 women. Standardized incidence ratio (SIR) with $95 \%$ confidence intervals (95\% CI) were calculated for each tumor. Results: A total of 1908 cases of GCT were identified. Seventy- nine $(4.14 \%)$ and $53(2.78 \%)$ patients were diagnosed with a malignant breast and uterine malignancy. The cumulative expected number of malignant breast and uterine tumors was $27(1.41 \%)$ and $6(0.31 \%)$, respectively. The calculated SIR for breast and uterine malignancies was $2.96(95 \% C I=2.34$, $3.68)$ and $8.83(95 \% C I=6.61,11.56)$, respectively. Conclusion: An increased incidence of breast and uterine malignancies among patients diagnosed with GCTs was observed.
\end{abstract}

Granulosa cell tumors (GCTs) are the most common malignant sex cord stromal tumors (SCST) of the ovary, comprising approximately $70 \%$ of all SCSTs (1). GCTs have an indolent course and an excellent prognosis when confined to the ovary $(2,3)$, with a five-year survival rate of $85-90 \%$ $(2,4)$. According to the National Cancer Institute's

Correspondence to: Dimitrios Nasioudis, MD, Division of Gynecologic Oncology, Department of Obstetrics and Gynecology, Hospital of the University of Pennsylvania, 3400 Spruce Street, 1 West Gates, Philadelphia, PA, 19104, U.S.A. Tel: +12156622730 , Fax: +1 215349-5224, e-mail: dimitrios.nasioudis@ uphs.upenn.edu

Key Words: Ovary, breast, granulosa cell tumor.
Surveillance, Epidemiology, and End Results (SEER) database, about $57 \%$ of women with GCTs present with stage I disease at the time of diagnosis and are amenable to primary surgical resection, usually with preservation of the uterus (5). On the genetic level, these tumors are known to be associated with a missense mutation in the FOXL2 gene, while there does not appear to be a hereditary component $(6,7)$.

Compared to other ovarian neoplasms, GCTs are hormonally active and are characterized by the secretion of estrogens, inhibins, and anti-Mullerian hormone $(2,6)$. Patients can, thus, present with signs of hyperestrogenemia such as precocious puberty, altered menstrual patterns, and postmenopausal bleeding (8), which can lead to an early detection of the tumors (9). In the setting of hyperestrogenemia, patients with GCTs frequently present with concurrent uterine pathology such as endometrial hyperplasia or carcinoma $(10,11)$. Several small studies have investigated the association between GCT and breast cancer, another estrogen-dependent malignancy (4, 5, 8-10). A recent population-based study from Finland demonstrated a significantly increased risk of breast cancer before the diagnosis of a GCT, providing more evidence to a shared physiology (7). Given the increased levels of circulating estrogens seen in patients with GCTs, our objective was to study the incidence of estrogen-related malignancies among women diagnosed with ovarian GCTs using the Surveillance, Epidemiology, and End Results (SEER) database, a large multi-institutional population-based database.

\section{Patients and Methods}

The US National Cancer Institute's SEER database was accessed and patients diagnosed between 1973 and 2014 with microscopically confirmed malignant ovarian GCTs (ICD-O-3 histology codes: 8620, $8621,8622)$ and had a known follow-up were selected. Personal tumor history was extracted for each patient and patients with a previous or subsequent malignant breast or uterine malignancy were identified. Clinico-pathological information was extracted from the de-identified SEER database. Given changes in ovarian cancer 
Table I. The incidence of breast cancer among patients diagnosed with ovarian granulosa cell tumors stratified by patient age at the end of follow-up.

\begin{tabular}{|c|c|c|c|c|c|c|c|}
\hline Age (yrs) & $\begin{array}{l}\text { Incidence } \\
\text { per } 100.000 \\
\text { women }^{1}\end{array}$ & $\begin{array}{c}\text { Incidence } \\
(\%)\end{array}$ & $\begin{array}{c}\text { Cummulative } \\
\text { incidence per } \\
100,000 \text { women }{ }^{2}\end{array}$ & $\begin{array}{l}\text { Cummulative } \\
\text { incidence } \\
(\%)\end{array}$ & $\begin{array}{c}\text { No. of } \\
\text { women } \\
\text { with GCT }\end{array}$ & $\begin{array}{l}\text { Expected no. of } \\
\text { women with } \\
\text { breast cancer }{ }^{3}\end{array}$ & $\begin{array}{c}\text { Observed } \\
\text { no }^{4}\end{array}$ \\
\hline $15-19$ & 0.2 & 0.0002 & 0.2 & 0.0002 & 24 & 0.000048 & 0 \\
\hline $20-24$ & 1.5 & 0.0015 & 1.7 & 0.0017 & 15 & 0.000255 & 0 \\
\hline $25-29$ & 9.2 & 0.0092 & 10.9 & 0.0109 & 32 & 0.003488 & 0 \\
\hline $30-34$ & 27.3 & 0.0273 & 38.2 & 0.0382 & 56 & 0.021392 & 0 \\
\hline $35-39$ & 60.8 & 0.0608 & 99 & 0.099 & 71 & 0.07029 & 2 \\
\hline $40-44$ & 122.7 & 0.1227 & 221.7 & 0.2217 & 115 & 0.254955 & 0 \\
\hline $45-49$ & 188.4 & 0.1884 & 410.1 & 0.4101 & 136 & 0.557736 & 1 \\
\hline $50-54$ & 224.7 & 0.2247 & 634.8 & 0.6348 & 196 & 1.244208 & 6 \\
\hline $55-59$ & 262.8 & 0.2628 & 897.6 & 0.8976 & 242 & 2.172192 & 4 \\
\hline $60-64$ & 338.6 & 0.3386 & 1236.2 & 1.2362 & 225 & 2.78145 & 7 \\
\hline $65-69$ & 424 & 0.424 & 1660.2 & 1.6602 & 198 & 3.287196 & 11 \\
\hline $70-74$ & 451.6 & 0.4516 & 2111.8 & 2.1118 & 155 & 3.27329 & 9 \\
\hline $75-79$ & 451.9 & 0.4519 & 2563.7 & 2.5637 & 165 & 4.230105 & 11 \\
\hline $80-84$ & 412.9 & 0.4129 & 2976.6 & 2.9766 & 118 & 3.512388 & 8 \\
\hline $85+$ & 343.5 & 0.3435 & 3320.1 & 3.3201 & 160 & 5.31216 & 20 \\
\hline Total & & & & & 1908 & 26.721153 & 79 \\
\hline
\end{tabular}

${ }^{1}$ Incidence per 100,000 women: age-specific rate of breast cancer in the general USA population as provided by the SEER database; ${ }^{2}$ Cummulative incidence per 100,000 women: sum of the incidence of this age group plus the incidence of the previous age groups; ${ }^{3}$ Expected number of women with breast cancer: calculated by multiplying cumulative incidence and number of patients with GCT in this age group; ${ }^{4}$ Observed number: number of patients diagnosed with breast cancer at the end of the follow-up in each age group.

staging over the study period, in order to avoid possible misclassifications, staging information was based on the SEERsummary stage. Localized stage includes tumors limited to the ovary, regional stage includes cancer that has spread to nearby lymph nodes or tissues while distant stage includes cases that have spread to distant lymph nodes or tissues. The expected incidence of breast and uterine cancer was calculated based on the US age-specific rates per 100,000 women, as provided by the SEER database. Patients were grouped based on their age at the end of follow-up to reflect the cumulative risk of breast and uterine cancer. Similar to previous studies, the cumulative expected cancer rate for each age group was calculated by adding the incidence of the previous age group (8). Standardized incidence ratio (SIR) with $95 \%$ confidence intervals was calculated for each tumor. The de-identified SEER data are available to the public for research purposes, while the present study was deemed exempt from the Institutional Board Review.

\section{Results}

A total of 1908 patients with GCTs who met the inclusion criteria were identified. The median age at the time of GCT diagnosis was 52 years (range $=3-94$ years). The majority were of White race $(73.1 \%)$ followed by Black $(19.9 \%)$, Asian/Pacific Islander (6.2\%) and Unknown race $(0.8 \%)$. Most patients had localized disease [1075 patients, $(56.3 \%)$ upon presentation], while $202(10.6 \%)$ and 480 (25.2\%) had regional and distant disease, respectively. For 151 patients, no staging information was available. The median follow-up of the present cohort was 83 months $(\mathrm{IQR}=130)$.
Seventy-nine $(4.14 \%)$ and $53(2.78 \%)$ patients had a personal history of a breast and uterine malignancy, respectively. The expected numbers of breast and uterine malignant tumors were $27(1.41 \%)$ and $6(0.31 \%)$ respectively, based on the US age-specific rates per 100,000 drawn from the SEER database. The calculated SIR for breast and uterine malignancies were $2.96(95 \% \mathrm{CI}=2.34,3.68)$ and 8.83 (95\% CI=6.61, 11.56). Tables I and II summarize the expected and observed incidence of breast and uterine malignancies among patients with ovarian granulosa cell tumors stratified by patient age.

The most common histologic subtype of uterine malignancy was endometrioid adenocarcinoma (52.8\%) (ICD-O-3 code $8380 ; 28$ patients), followed by adenocarcinoma NOS $(35.8 \%)$ (ICD-O-3 code $8140 ; 20$ patients) and other histologies (6 patients). For all but 2 patients the GCT and uterine malignancies were diagnosed concurrently (within 6 months from each other). Median patient age at uterine malignancy diagnosis was 58 years (range $=36-82$ years), while $84.9 \%$ had disease localized to the uterus.

A total of 87 primary breast tumors were observed in 79 patients. The median patient age at the time of breast cancer diagnosis was 66 years (range $=30-87$ years). In 26 patients $(32.9 \%)$ breast malignancy preceded the diagnosis of GCT with a median interval between breast and ovarian malignancy diagnosis of 91.3 months (range=10-341 months). For seven patients, breast and GCT were diagnosed 
Table II. The incidence of uterine malignancies among patients diagnosed with ovarian granulosa cell tumors stratified by patient age at the end of follow-up.

\begin{tabular}{|c|c|c|c|c|c|c|c|}
\hline Age (yrs) & $\begin{array}{l}\text { Incidence } \\
\text { per } 100.000 \\
\text { women }^{1}\end{array}$ & $\begin{array}{c}\text { Incidence } \\
(\%)\end{array}$ & $\begin{array}{c}\text { Cumulative } \\
\text { incidence per } \\
100,000 \text { women }^{2}\end{array}$ & $\begin{array}{l}\text { Cumulative } \\
\text { incidence } \\
(\%)\end{array}$ & $\begin{array}{c}\text { No. of } \\
\text { women } \\
\text { with GCT }\end{array}$ & $\begin{array}{l}\text { Expected no. of } \\
\text { women with } \\
\text { uterine cancer }{ }^{3}\end{array}$ & $\begin{array}{c}\text { Observed } \\
\text { no }\end{array}$ \\
\hline $15-19$ & 0.1 & 0.0001 & 0.1 & 0.0001 & 24 & 0.000024 & 0 \\
\hline $20-24$ & 0.15 & 0.00015 & 0.25 & 0.00025 & 15 & 0.0000375 & 0 \\
\hline $25-29$ & 2 & 0.002 & 2.25 & 0.00225 & 32 & 0.00072 & 0 \\
\hline $30-34$ & 4.9 & 0.0049 & 7.15 & 0.00715 & 56 & 0.004004 & 0 \\
\hline $35-39$ & 9.2 & 0.0092 & 16.35 & 0.01635 & 71 & 0.0116085 & 0 \\
\hline $40-44$ & 15.2 & 0.0152 & 31.55 & 0.03155 & 115 & 0.0362825 & 2 \\
\hline $45-49$ & 24.8 & 0.0248 & 56.35 & 0.05635 & 136 & 0.076636 & 1 \\
\hline $50-54$ & 45.6 & 0.0456 & 101.95 & 0.10195 & 196 & 0.199822 & 0 \\
\hline $55-59$ & 76.4 & 0.0764 & 178.35 & 0.17835 & 242 & 0.431607 & 9 \\
\hline $60-64$ & 94.8 & 0.0948 & 273.15 & 0.27315 & 225 & 0.6145875 & 15 \\
\hline $65-69$ & 106.2 & 0.1062 & 379.35 & 0.37935 & 198 & 0.751113 & 4 \\
\hline $70-74$ & 97.8 & 0.0978 & 477.15 & 0.47715 & 155 & 0.7395825 & 3 \\
\hline $75-79$ & 86 & 0.086 & 563.15 & 0.56315 & 165 & 0.9291975 & 10 \\
\hline $80-84$ & 70 & 0.07 & 633.15 & 0.63315 & 118 & 0.747117 & 7 \\
\hline \multirow[t]{2}{*}{$85+$} & 53 & 0.053 & 686.15 & 0.68615 & 160 & 1.09784 & 2 \\
\hline & & & & & 1908 & 5.640179 & 53 \\
\hline
\end{tabular}

${ }^{1}$ Incidence per 100,000 women: age-specific rate of uterine cancer in the general US population as provided by the SEER database; ${ }^{2}$ Cumulative incidence per 100,000 women: sum of the incidence of this age group plus the incidence of the previous age groups; ${ }^{3}$ Expected number of women with uterine cancer: calculated by multiplying cumulative incidence and number of patients with GCT in this age group; ${ }^{4}$ Observed number: number of patients diagnosed with uterine cancer at the end of the follow-up in each age group.

concurrently (within 6 months from each other). Forty-six patients developed a breast malignancy following the diagnosis of GCT with a median interval of 120.8 months (range $=8-372$ months). The most common histologic subtype was infiltrating ductal carcinoma (67.8\%) (ICD-O-3: 8500, 59 cases) and $72.4 \%$ of cases were of localized stage. Based on available information, $79.7 \%$ (47/59) and 61\% (36/59) of tumors were estrogen and progesterone receptor positive respectively. When excluding patients who developed a breast cancer before or concurrently with the diagnosis of a GCT, calculated SIR was $1.72(95 \% \mathrm{CI}=1.26,2.29)$.

\section{Discussion}

In the largest cohort of patients with GCT to date, $4.14 \%$ had a personal history of breast cancer and $2.78 \%$ had a diagnosis of endometrial cancer. Our study thus provides further compelling evidence for an association between GCT and estrogen-related malignancies.

Several smaller studies prior to this population-based study have provided insight into the association between GCTs and breast and endometrial neoplasms. Historically, Evans et al. found a $5.5 \%$ incidence of breast cancer in 200 women with GCTs or theca cell tumors (9). Ohel et al. reported a $6.4 \%$ incidence of breast cancer among 172 patients diagnosed with GCT after a follow-up of 15-years, approximately 4 times higher than the incidence in the general population (10). An $11 \%$ incidence of endometrial pathology was also found among the 63 patients who had endometrial examination (10). Of note, this study did not differentiate between GCTs and theca cell tumor patients (10). Björkholm et al. analyzed the Swedish Cancer Registry and identified patients with GCT or theca-cell tumors diagnosed between 1958 and 1972. A total of 15 patients had a prior diagnosis of breast cancer, while four patients were concurrently diagnosed with breast cancer and GCT. The expected number of breast malignancies in that cohort was 10.3 with a calculated relative risk of $1.8(95 \% \mathrm{CI}=1.1,2.9)$. Authors also found a four times increased risk of developing an endometrial carcinoma compared to the lifetime risk of the general population (12). In a more recent populationbased study, Bryk et al. used the Finnish Cancer Registry to determine the incidence of new primary malignancies after GCTs, as well as the incidence of GCT following breast cancer. They identified 986 patients diagnosed between 1968 and 2013 and found breast cancer rate of $6.9 \%$. An increased risk of developing breast cancer was only significant for malignancies diagnosed prior to the diagnosis of GCTs; they suggested that the lack of significance of developing breast cancer after a primary GCTs was due to the presence of surveillance bias. Interestingly, in this cohort the rate of uterine cancer was $2.5 \%$ lower than that previously reported, 
possibly due to the inclusion of a large number of women with a prior hysterectomy (7). Hammer et al., in a retrospective study, analysed 163 women diagnosed with a GCT between 1962 and 2003 at hospitals in Jutland, Denmark and found an increased risk of breast cancer $(\mathrm{OR}=3.3,95 \% \mathrm{CI}=1.6,6.6)$ compared to the general population (8). Meisel et al. in a secondary analysis of the data of 118 patients that received chemotherapy for any stage GCT, found that $12(10 \%)$ had a diagnosis of breast cancer prior to the diagnosis of GCT, an incidence that was 3.22 times higher than that expected based on the SEER rates (5). Van Meurs et al. analyzed a cohort of 1031 patients with GCT derived from the PALGA database of the Netherlands (11). For 614 patients, endometrial tissue was available for examination at the time of initial GCT diagnosis; $58(5.9 \%)$ patients were found to have endometrial cancer (11). For 490 patients who did not undergo hysterectomy during the initial surgery for GCT, no increased risk of endometrial cancer was observed since only two $(0.4 \%)$ patients were diagnosed with endometrial cancer after approximately 10 years of follow-up (11). Interestingly both patients had recurrent GCT at the time of endometrial cancer diagnosis (11).

The relationship between GCTs and estrogen-related malignancies could be explained physiologically through hyperestrogenism stimulating malignant breast tumor growth and endometrial hyperplasia. Interestingly, in Meisel et al.'s study (5), $100 \%(n=5)$ of the breast tumors identified in GCTs patients stained positive for estrogen receptor (ER) and progesterone receptor (PR). However, in the current study, $79.7 \%(47 / 59)$ and $61 \%(36 / 59)$ of tumors were estrogen and progesterone receptor positive respectively, comparable to the rate of hormone receptor positive breast cancer found in the general US population (13). Potentially, increased levels of estrogen in patients with GCT may regulate the differentiation of mammary stem cells located at niches in the basal epithelium contributing to breast cancer tumorigenesis (14). The associations with parity and obesity in patients also provide plausible hormonal similarities. However, a common yet unknown genetic susceptibility cannot be excluded as a causal etiology. For now, BRCAl and $B R C A 2$ genes, associated with breast cancer, are not known to be associated with GCTs and the pathognomic FOXL2 gene of GCTs is not known to be associated with breast cancer $(2,6)$. Therefore, metabolites of tamoxifen may contribute to the development of GCTs in breast cancer survivors (14).

The limitations of the present study include the lack of central pathology review and the loss during follow-up due to patient migration out of a SEER county. Our study is also limited by the inclusion of both previous, concomitant, and subsequent breast and uterine cancers, such that direct causal and temporal relationships cannot be drawn. However, even when excluding breast tumors diagnosed prior to GCT, patients had an increased risk of breast cancer compared to the general population. Use of tamoxifen for breast cancer could not be extrapolated from the data to measure the impact on risk. Moreover, certain confounding factors such as parity, BMI, use of hormone therapy and presence of premalignant breast lesions are not available in the SEER database. The strengths of the present data include the use of a multi-institutional racially diverse population-based database and the large sample size with a long follow-up.

Despite the limitations of our data, in this populationbased study, an increased risk of uterine and breast malignancies was observed among women with ovarian GCTs. This information provides further evidence to a strong relationship between GCTs and the risk of developing breast or endometrial malignancies and could guide screening strategies and surgical planning of these patients in the future. We also suggest the creation of an international tumor registry of GCTs to further elucidate the optimal management of these patients. With greater numbers of patients, definitive analyses can be appropriately drawn to guide treatment and surveillance guidelines for these women. Patients diagnosed with GCT may benefit from earlier breast cancer screening and close follow-up.

\section{Conflicts of Interest}

The Authors have no conflicts of interest to declare regarding this study.

\section{Authors' Contributions}

DN: conception, statistical analysis, critical analysis, drafting/final editing; EW, SM, GS, AH, EK: critical analysis, drafting/final editing; NL: supervision, statistical analysis, critical analysis, drafting/final editing.

\section{References}

1 Colombo N, Parma G, Zanagnolo V and Insinga A: Management of ovarian stromal cell tumors. J Clin Oncol 25(20): 2944-2951, 2007. PMID: 17617526 . DOI: 10.1200/JCO.2007.11.1005

2 Schumer ST and Cannistra SA: Granulosa cell tumor of the ovary. J Clin Oncol 21: 1180-1189, 2003. PMID: 12637488. DOI: $10.1200 / \mathrm{JCO} .2003 .10 .019$

3 Nasioudis D, Kanninen TT, Holcomb K, Sisti G and Witkin SS: Prevalence of lymph node metastasis and prognostic significance of lymphadenectomy in apparent early-stage malignant ovarian sex cord-stromal tumors. Gynecol Oncol 145(2): 243-247, 2017. PMID: 28292524. DOI: 10.1016/j.ygyno.2017.03.005

4 Björkholm E: Granulosa cell tumors: a comparison of survival in patients and matched controls. Am J Obstet Gynecol 138: 329-331, 1980. PMID: 7416223. DOI 10.1016/0002-9378(80) 90257-4

5 Meisel JL, Hyman DM, Jotwani A, Zhou Q, Abu-Rustum NR, Iasonos A, Pike MC and Aghajanian C: The role of systemic chemotherapy in the management of granulosa cell tumors. 
Gynecol Oncol 136(3): 505-511, 2015. PMID: 25546114. DOI: 10.1016/j.ygyno.2014.12.026

6 Jamieson S, Butzow R, Andersson N, Alexiadis M, UnkilaKallio L, Heikinheimo M, Fuller PJ and Anttonen M: The FOXL2 C134W mutation is characteristic of adult granulosa cell tumors of the ovary. Mod Pathol 23: 1477-1485, 2010. PMID: 20693978. DOI: 10.1038/modpathol.2010.145

7 Bryk S, Pukkala E, Färkkilä A, Heikinheimo M, Unkila-Kallio L and Riska A: Other primary malignancies among women with adult-type ovarian granulosa cell tumors. Int J Gynecol Cancer 28(8): 1529-1534, 2018. PMID: 30036228. DOI: 10.1097/ IGC.0000000000001333

8 Hammer A, Lauszus FF and Peterson AC: Ovarian granulosa cell tumor and increased risk of breast cancer. Acta Obstet Gynecol Scand 92(12): 1422-1425, 2013. PMID: 30036228. DOI: $10.1097 /$ IGC.0000000000001333

9 Evans AT 3rd, Gaffey TA, Malkasian GD Jr and Annegers JF: Clinicopathologic review of 118 granulosa and 82 tehca cell tumors. Obstet Gynecol 55: 231-238, 1980. PMID: 6243409.

10 Ohel G, Kaneti H and Schenker JG: Granulosa cell tumors in Israel: A study of 172 cases. Gynecol Oncol 15: 278-286, 1983. PMID: 6832637.
11 van Meurs HS, Bleeker MC, van der Velden J, Overbeek LI, Kenter GG and Buist MR: The incidence of endometrial hyperplasia and cancer in 1031 patients with a granulosa cell tumor of the ovary: long-term follow-up in a population-based cohort study. Int J Gynecol Cancer 23: 1417-1422, 2013. PMID: 24257556. DOI: $10.1097 /$ IGC.0b013e3182a57fb4

$12 \mathrm{Li} \mathrm{CI}$, Daling JR, and Malone KE: Incidence of invasive breast cancer by hormone receptor status from 1992 to 1998 . J Clin Oncol 21(1): 28-34, 2003. PMID: 12506166. DOI: 10.1200/JCO. 2003.03.088

13 Zolfaroli I, Tarín JJ and Cano A: The action of estrogens and progestogens in the young female breast. Eur J Obstet Gynecol Reprod Biol 230: 204-207, 2018. PMID: 29627127. DOI: 10.1016/j.ejogrb.2018.03.057

14 Gherman RB, Parker MF and Macri CI: Granulosa cell tumor of the ovary associated with antecedent tamoxifen use. Obstet Gynecol 84: 717-719, 1994. PMID: 9205462.

Received July 18, 2019

Revised August 3, 2019

Accepted August 5, 2019 